\title{
The rationale for ISO 14001 certification: A systematic review and a cost- benefit analysis
}

Mark Anthony Camilleri, University of Malta', Malta and University of Edinburgh, Scotland.

This is a prepublication version.

Suggested Citation: Camilleri, M.A. (2022). The rationale for ISO 14001 certification: A systematic review and a cost-benefit analysis, Corporate Social Responsibility and Environmental Management, https://doi.org/10.1002/csr.2254

\begin{abstract}
This contribution presents the findings from a two-stage systematic review. It relied on PRISMA's methodical protocol to capture and analyze high-impact articles, that were focused on the International Standards Organization's ISO 14001 - Environment Management Systems. Whilst stage 1 shed light on the most cited publications since 1995, stage 2 narrowed down the search results between 2015 and 2021. The findings suggest that the use of this certifiable standard may result in operational efficiencies through better utilization of resources and waste management systems. It provides opportunities for practitioners to re-conceive their license to operate and to enhance their credentials with stakeholders. It considered potential pitfalls like high certification costs, time constraints as well as an increase in paperwork and red tape. Moreover, it recognizes that managers and employees may not be willing to implement changes as they may prefer the status quo or could not be knowledgeable enough to integrate the standard's environment management systems with existing practices.
\end{abstract}

Keywords: Environment management; ISO 14001; environmental performance; cost-benefit analysis; systematic review; PRISMA.

\footnotetext{
${ }^{1}$ Department of Corporate Communication, Faculty of Media and Knowledge Sciences, University of Malta, Malta. Email: mark.a.camilleri@um.edu.mt
} 


\section{Introduction}

During the Paris Climate Conference (COP 21), one hundred ninety-six (196) countries pledged their commitment to implement environmental performance measures to reduce the effects of climate change. This conference has led to the development of the 'Paris Agreement' where signatories became legally bound to limit global warming to below $2{ }^{\circ} \mathrm{C}$, and possibly $1.5^{\circ} \mathrm{C}$ (Palea \& Drogo, 2020; Secinaro, Brescia, Calandra \& Saiti, 2020). They recognized the importance of averting and minimizing the environmental impact that is caused by climate change, by scaling up their efforts and support initiatives to reduce emissions, by building resilience among parties, and by promoting cooperation (Birindelli \& Chiappini, 2021; Gatto, 2020).

In the aftermath of COP 21, many countries submitted their plans for climate action (these plans are also known as nationally determined contributions - NDCs), where they communicated about their tangible actions that were aimed to reduce their greenhouse gas emissions and the impacts of rising temperatures (Fatica \& Panzica, 2021; Gerged, Matthews \& Elheddad, 2021). Consequentially, intergovernmental organizations including the European Union (EU), among others, are increasingly establishing ambitious carbon neutrality goals and zero-carbon solutions to tackle climate change issues (Benz, Paulus, Scherer, Syryca \& Trück, 2021).

Many countries are incentivizing businesses across different economic sectors, to reduce their emissions. For example, the EU member states are expected to reduce their greenhouse gas emissions by $40 \%$ before 2030, and by $60 \%$ prior to 2050 (EU, 2019). These targets would require the commitment of stakeholders from various sectors including those operating within the energy and transportation industries, among others.

The latest climate change conference (COP26) suggested that progress has been made on the signatories' mitigation measures that were aimed to reduce emissions, on their adaptation efforts to deal with climate change impacts, on the mobilization of finance, and on the increased 
collaboration among countries to reach 2030 emissions targets. However, more concerted efforts are required to deliver on these four pledges (UNFCC, 2021).

This contribution raises awareness on the use of environmental management standards that are intended to support organizations of different types and sizes, including private entities, not-for-profits as well as governmental agencies, to improve their environmental performance credentials. A thorough review of the relevant literature suggests that, over the years many practitioners have utilized the International Standards Organization's ISO 14001 environment management systems standard to assist them in their environmental management issues (Baek, 2018; Delmas \& Toffel, 2008; Erauskin-Tolosa, Zubeltzu-Jaka, Heras-Saizarbitoria \& Boiral, 2020; Melnyk, Sroufe \& Calantone, 2003).

Many academic commentators noted that several practitioners operating in different industry sectors, in various contexts, are implementing ISO 14001 requirements to obtain this standard's certification (Boiral, Guillaumie, Heras-Saizarbitoria \& Tayo Tene, 2018; ParaGonzález \& Mascaraque-Ramírez, 2019; Riaz, \& Saeed, 2020). Whilst several researchers contended about the benefits of abiding by voluntary principles and guidelines (Camilleri, 2018), others discussed about the main obstacles to obtaining impartial audits, assurances and certifications from independent standard setters (Hillary, 2004; Ma, Liu, Appolloni \& Liu, 2021; Robèrt, Schmidt-Bleek, Aloisi De Larderel ... \& Wackernagel, 2002; Teng \& Wu, 2018).

Hence, this research examines identifies the rationale for ISO 14001 certification (Carvalho, Santos \& Gonçalves, 2020; Eltayeb, Zailani \& Ramayah, 2011; Lee, Noh, Choi \& Rha, 2017; Potoski \& Prakash, 2005) that is supposedly intended to improve the organizations' environmental performance and to enhance their credentials. Specifically, this contribution's objectives are threefold. Firstly, it provides a generic background on voluntary instruments, policies and guidelines that are intended to promote corporate environmentally responsible behaviors. Secondly, it presents the results from a systematic review of academic articles that were 
focused on ISO 14001 - environment management systems. Thirdly, it synthesizes the findings from high impact papers and discusses about the benefits and costs of using this standard. In conclusion, it elaborates on the implications of this research, it identifies its limitations and points out future research avenues.

In sum, this contribution differentiates itself from previous articles, particularly those that sought to investigate the introduction and implementation of environment management systems in specific entities. This research involves a two-stage systematic analysis. It appraises a number of empirical investigations, theoretical articles, reviews, case studies, discursive/opinion papers, from 1995-2021. Afterwards, it scrutinizes their content to shed more light on the pros and cons of using ISO 14001 as a vehicle to improve corporate environmental performance.

\section{Regulatory instruments, principles and guidelines for good practice}

Stakeholders expect organizations including businesses, governmental organizations as well as non-government organizations (NGOs), to do the right things and to communicate about their laudable activities. They must be seen operating in a responsible manner, by looking after their employees, by taking care of the environment, and by being good corporate citizens. When problems occur, businesses must act quickly to resolve problematic matters. While many governments and their policymakers have been the primary force for the promotion of financial reporting standards (Camilleri, 2017a; Weber, 2014), intergovernmental institutions like the United Nations Global Compact, the Organization for Economic Cooperation and Development (OECD) as well as a number of NGOs, including ISO, the Global Reporting Initiative (GRI) and the International Integrated Reporting Council (IIRC), among others, have 
increasingly facilitated the growth and diffusion of non-financial reporting mechanisms (Camilleri, 2017b; Fortuna, Testarmata, Sergiacomi \& Ciaburri, 2020).

Several NGOs developed predefined norms, procedures and guidelines to support organizations, including businesses, on social, environmental management and corporate governance issues (Boström \& Hallström, 2010; Salomone, 2008). For example, ISO developed performance and/or process-oriented standards that can be applied across different industry sectors and geographic regions (Dissou, 2005). A few substantive standards can also provide certification for compliance as well as independent monitoring systems and assurance mechanisms (Husted, Montiel \& Christmann, 2016).

However, in many cases, certifiable standards are adopted on a voluntary basis. They are considered as soft law solutions, as they are not legally-binding, although they may fill numerous governance and accountability gaps for which there is no applicable regulation or enforcement (Nakamura, Takahashi \& Vertinsky, 2001). Their principles may eventually be internalized by practitioners as they allow them to improve their credentials with stakeholders (Husted et al., 2016) and to meet the expectations of society.

The organizations' stakeholders can make or break their reputation and standing (Park, 2019). Positive as well as adverse publicity that is generated through media events or during public protests can impact directly on corporate image and on revenues (Xu, Zeng, Zou \& Shi, 2016). They can indirectly affect share prices and access to capital investment ( $\mathrm{Su}$, Swanson \& Chen, 2018).

Consumers can impose direct economic costs on non-compliant companies by boycotting products or services in addition to negative word-of-mouth marketing. Stakeholders including the governments, regulatory authorities, NGOs and other organizations within the supply chain, may severely sanction irresponsible organizations if they do not respect societal norms and ethical values (Camilleri, 2016). Bad publicity could induce firms to adopt beyond-compliance 
measures to engage in corporate citizenship (Wilburn \& Wilburn, 2011). Arguably, private businesses are continuously expected to prove their social license to operate, and to avoid unnecessary criticism from stakeholders (Colwell \& Joshi, 2013; Demuijnck \& Fasterling, 2016).

The institutional theory posits that firms ought to respond to regulatory pressures in society (Clemens \& Douglas, 200). They have to do so to access critical resources or merely for social acceptance purposes (Ntim \& Soobaroyen, 2013). The organizations' compliance with their operating environment's regulations enables them to improve their legitimacy among stakeholders (Beck, Dumay \& Frost, 2017).

Hence, there is scope for them to anticipate hard legislation. They could impose stricter regulations on themselves to prevent irregularities, and/or to reduce their chances of legal violations (Hillary, 2004). If they do so, they will be perceived as credible and trustworthy. As a result, they would be in a better position to build their reputation, and to achieve a competitive advantage relative to their rivals (Khan, Yang \& Waheed, 2019; Orsato, 2006; Yu, Kuo \& Kao, 2017). Businesses can enhance their legitimacy with stakeholders by communicating about their non-financial performance (Balluchi, Lazzini \& Torelli, 2021). They can disclose information on their corporate governance, social and environmentally sound behaviors in corporate social responsibility (CSR) or Sustainability reports (Duthler \& Dhanesh, 2018; Ettinger, 2018).

The American Institute of Certified Public Accountants' Jenkins Report may be considered as one of the major documents that has provided the foundations for non-financial disclosures. Other guidelines that are focused on environmental management systems were developed by intergovernmental agencies as well as by standard setters (including ISO), to assist businesses and other organizations to improve their environmental responsibility credentials (Romito \& Vurro, 2021). 
For example, the EU's non-financial reporting directive (NFRD) law requires that large undertakings including corporations, listed businesses and government entities, among others, to disclose information on the way they operate and manage social and environmental challenges (Camilleri, 2015a). This helps investors, civil society organizations, consumers, policy makers and other stakeholders to be in a better position to evaluate the companies' non-financial performance (EU, 2014).

Recently, EU (2021) put forward its proposal for a Corporate Sustainability Reporting Directive (CSRD), which shall amend the existing reporting requirements of the EU's NFRD. In sum, this proposal extends the audit requirement to large entities including government agencies, listed businesses, financial organizations like banks and insurance companies, among others, in regulated markets (except listed micro-enterprises). They will be expected to introduce more detailed reporting requirements, according to mandatory EU sustainability reporting standards. At the time of writing this paper, it is envisaged that the first set of standards could be adopted by October 2022 (EU, 2021).

Regulatory principles and guidelines can help practitioners to identify, manage, monitor and control corporate environmental issues in a "holistic" manner (Melnyk et al., 2003). Such instruments and substantive systems are meant to support organizations in their waste management, and in reducing pollution, while simultaneously improving their environmental and financial performance (Camilleri, 2021a; Rivera-Arrubla, Zorio-Grima \& García-Benau, 2017).

For example, ISO 14000 family of standards as well as the European Union's Eco-Management and Audit Scheme (EMAS) cover different aspects that promote greener environmental management performance. EMAS raises awareness on sustainable energy generation capacities through renewable sources, regular assessments on the co-generation of heating facilities, periodic material disclosures and monitoring on energy efficiencies, et cetera. Moreover, ISO 
standards are purposely devised to address perennial issues including greenhouse gas emissions, water and air pollution prevention, among others.

\subsection{ISO family of standards}

ISO 14000 family of environmental management standards (EMSs) are developed by the International Standards Organization's Technical Committee ISO/TC 207 and its various subcommittees. They comprise principles and guidelines to help organizations to: (i) minimize their impact on the environment, (ii) comply with relevant legislation and regulations, and (c) to foster continuous operational improvements in their environmental management practices. ISO 14001 - Environmental management systems - Requirements with guidance for use (October 2015) is a voluntary, certifiable standard that specifies the requirements for an effective environmental management system.

This standard encourages practitioners toward achieving continuous improvements in their organizational approaches to minimize their impact on the environment (Klassen \& Vachon, 2003; Kitazawa \& Sarkis, 2000). It recommends that environmental management issues ought to be embedded within the organizations' strategic planning processes (Delmas, 2001, Eltayeb, Zailani \& Ramayah, 2011). ISO 14001 specifies that leaders have to pledge their commitment to implement sustainable initiatives, to protect the environment and to mitigate climate change (Bravi, Santos, Pagano \& Murmura, 2020; Chiarini, 2019). This standard raises awareness on the importance of promoting corporate environmentally responsible behaviors through different media and of forging relationships with different stakeholders. A number of other ISO standards are focused on specific aspects of the environment, including ISO 4226, ISO 6107 and ISO 10381, among others. Table 1 provides a non-exhaustive list of ISO's environmental standards. 
Table 1. A list of ISO environmental standards

\begin{tabular}{|c|c|}
\hline ISO 4226 & Air Quality \\
\hline ISO 6107 & Water Quality \\
\hline ISO 10381 & Soil quality - Sampling \\
\hline
\end{tabular}

\begin{tabular}{|c|c|}
\hline ISO 14001 & Environmental management systems - Requirements with guidance for use \\
\hline ISO 14002 & $\begin{array}{l}\text { Environmental management systems - Guidelines for using ISO } 14001 \text { to ad- } \\
\text { dress environmental aspects and conditions within an environmental topic area } \\
\text { - Part 1: General }\end{array}$ \\
\hline ISO 14004 & Environmental management systems - General guidelines on implementation \\
\hline ISO 14005 & $\begin{array}{l}\text { Environmental management systems - Guidelines for a flexible approach to } \\
\text { phased implementation }\end{array}$ \\
\hline ISO 14006 & Environmental management systems - Guidelines for incorporating eco-design \\
\hline ISO 14015 & $\begin{array}{l}\text { Environmental management - Environmental assessment of sites and organi- } \\
\text { zations (EASO) }\end{array}$ \\
\hline ISO 14020 & Environmental labels and declarations - General principles \\
\hline ISO 14021 & $\begin{array}{l}\text { Environmental labels and declarations: Self-declaration of environmental } \\
\text { claims: guidelines, definitions and usage of terms }\end{array}$ \\
\hline ISO14024 & $\begin{array}{l}\text { Environmental labels and declarations - Type I Environmental labelling - Prin- } \\
\text { ciples and procedures }\end{array}$ \\
\hline ISO 14030 & $\begin{array}{l}\text { Environmental performance evaluation - Green debt instruments - Part 1: } \\
\text { Process for green bonds }\end{array}$ \\
\hline ISO 14031 & $\begin{array}{l}\text { Environmental management - Environmental performance evaluation - Guide- } \\
\text { lines }\end{array}$ \\
\hline ISO 14040 & $\begin{array}{l}\text { Environmental management - Life cycle assessment - Principles and frame- } \\
\text { work }\end{array}$ \\
\hline ISO 14044 & $\begin{array}{l}\text { Environmental management - Life cycle assessment - Requirements and guide- } \\
\text { lines }\end{array}$ \\
\hline ISO 14050 & Environmental management - Vocabulary \\
\hline ISO 14062 & $\begin{array}{l}\text { Environmental management - Integrating environmental aspects into product } \\
\text { design and development }\end{array}$ \\
\hline ISO 14063 & $\begin{array}{l}\text { Environmental management - Environmental communication - Guidelines and } \\
\text { examples }\end{array}$ \\
\hline ISO 14064 & $\begin{array}{l}\text { Greenhouse gases; measuring, quantifying, and reducing greenhouse gas emis- } \\
\text { sions }\end{array}$ \\
\hline ISO 14065 & $\begin{array}{l}\text { General principles and requirements for bodies validating and verifying envi- } \\
\text { ronmental information }\end{array}$ \\
\hline ISO 14067 & $\begin{array}{l}\text { Greenhouse gases - Carbon footprint of products - Requirements and guide- } \\
\text { lines for quantification }\end{array}$ \\
\hline ISO 14090 & Adaptation to climate change - Principles, requirements and guidelines \\
\hline ISO 15099 & Thermal performance of windows, doors and shading devices \\
\hline ISO 15270 & Plastics - Guidelines for the recovery and recycling of plastics waste \\
\hline ISO 17029 & Conformity assessment \\
\hline ISO 50001 & stem \\
\hline
\end{tabular}




\section{Methodology}

This research adopted PRISMA’s robust protocol (Moher, Shamseer, Clarke, Ghersi, Liberati, Petticrew ... \& Stewart, 2015) to systematically extract and scrutinize the content from academic sources that were indexed in Scopus. Hence, this review was carefully planned and documented in all stages, to ensure that its accountability, integrity, and transparency. The search query considered only those articles that were published in peer-reviewed journals. The contributions that that were published in books, book series, conference proceedings and trade journals were excluded from this analysis - as they were not considered as rigorous as academic journals (Boiral et al., 2018).

Scopus featured a list of contributing authors and identified their articles' titles, publisher, subject areas and keywords. The researcher sorted the publications from highest to lowest number of citations. Figure 1 clarifies the methodology that was used to capture, analyze and synthesize the findings for this systematic review. 
Figure 1: The PRISMA protocol that was used for this systematic review

Formulation of the research question

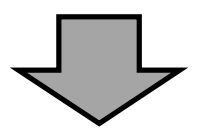

Systematic analysis - Stage 1

Relevant keywords were inserted in the search query.

Only contributions that were published in (Scopus-indexed) journals were identified and screened in this review.

The query yielded a list of top journals, subject areas of previous articles and reported the most popular keywords that were inserted by the contributing authors.

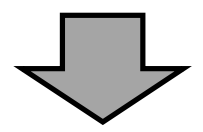

Systematic analysis - Stage 2

A similar methodology was used as in Stage 1. The review was limited to articles and reviews from journals that were published in the last 7 years, specifically between January 2015 - December 2021.

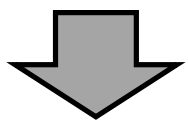

Syntheses and interpretation of the findings from eligible contributions

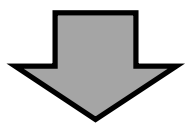

\section{Conclusions and implications}

The systematic review considered publications that featured "ISO 14001" in their title, abstract and keywords, as of January 2022. The search revealed that there were 2,273 contributions since 1995 .

According to the systematic analysis (stage 1), there were 1,367 journal articles that were written in English. According to Scopus, the top 10 subject areas of these articles were related to Environmental Science (667); Business, Management and Accounting (661); Engineering 
(436); Social Sciences (308); Energy (240); Economics, Econometrics and Finance (125); Medicine (83), Agriculture and Biological Sciences (63), and Materials Science (57).

The Journal of Cleaner Production has published 151 articles on this topic. It was followed by Business Strategy and the Environment (39); Environmental Quality Management (38); Sustainability Switzerland (28); Corporate Social Responsibility and Environmental Management (27); Corporate Environmental Strategy (22); Journal of Environmental Management (22); Greener Management International (19); Management of Environmental Quality: An International Journal (19), and Quality Progress (16).

Over time, the most used keywords included: ISO 14001 (505); Environmental Management (452); Environmental Management Systems (243); Sustainable Development (155); Standards (146); Environmental Protection (143); Environmental Management System (142); Environmental Impact (130), Certification (119) and Sustainability (109).

Table 2 presents a list of 35 most-cited articles on "ISO 14001" that accumulated more than 200 citations in Scopus (to date). It endorses the contributing authors, describes their methodological approaches, and features the keywords of their research. 
Table 2. List of most-cited academic articles on ISO 14001

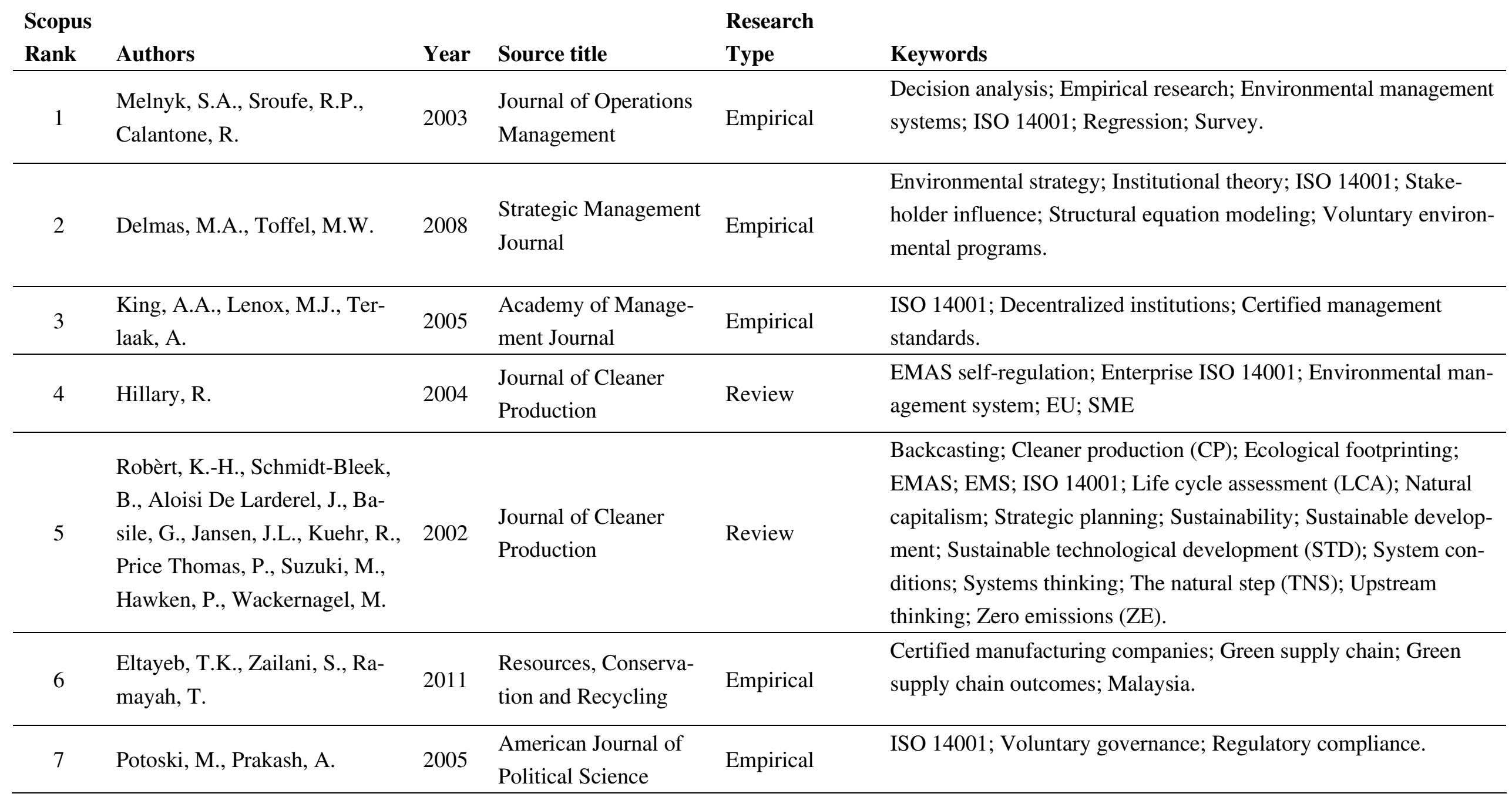




\begin{tabular}{|c|c|c|c|c|c|}
\hline 8 & Boiral, O. & 2007 & Organization Science & Case study & $\begin{array}{l}\text { Environmental performances; ISO 14001; Organizational hypoc- } \\
\text { risy; Rational myths; Ritual integration; Social legitimacy. }\end{array}$ \\
\hline 9 & $\begin{array}{l}\text { Jacobs, B.W., Singhal, V.R., } \\
\text { Subramanian, R. }\end{array}$ & 2010 & $\begin{array}{l}\text { Journal of Operations } \\
\text { Management }\end{array}$ & Empirical & $\begin{array}{l}\text { Awards; Certifications; Environmental initiatives; Environmental } \\
\text { performance; Market value; Stock market reaction. }\end{array}$ \\
\hline 10 & Klassen, R.D., Vachon, S. & 2003 & $\begin{array}{l}\text { Production and Opera- } \\
\text { tions Management }\end{array}$ & Empirical & $\begin{array}{l}\text { Environmental management; ISO 14001; Pollution control; Pollu- } \\
\text { tion prevention; Supply chain management. }\end{array}$ \\
\hline 11 & Delmas, M.A. & 2002 & Policy Sciences & Review & $\begin{array}{l}\text { Environmental management; Industrial regulation; Institutional } \\
\text { framework; Standard; Waste management. }\end{array}$ \\
\hline 12 & Morrow, D., Rondinelli, D. & 2002 & $\begin{array}{l}\text { European Manage- } \\
\text { ment Journal }\end{array}$ & Case study & $\begin{array}{l}\text { EMAS; EMS; Energy; Environment; Environmental manage- } \\
\text { ment; Gas; ISO } 14001 \text {. }\end{array}$ \\
\hline 13 & $\begin{array}{l}\text { Rennings, K., Ziegler, A., An- } \\
\text { kele, K., Hoffmann, E. }\end{array}$ & 2006 & Ecological Economics & Empirical & $\begin{array}{l}\text { Economic performance; Environmental innovations; Environmen- } \\
\text { tal management systems; Sustainability. }\end{array}$ \\
\hline 14 & Orsato, R.J. & 2006 & $\begin{array}{l}\text { California Manage- } \\
\text { ment Review }\end{array}$ & Discursive & $\begin{array}{l}\text { Competitive advantage; Competitive environmental strategies; Eco } \\
\text { efficiency; Beyond compliance leadership; Eco-branding; Environ- } \\
\text { mental cost leadership. }\end{array}$ \\
\hline 15 & Bansal, P., Hunter, T. & 2003 & $\begin{array}{l}\text { Journal of Business } \\
\text { Ethics }\end{array}$ & Empirical & $\begin{array}{l}\text { Early adoption; ISO 14001; Strategic realignment; Strategic rein- } \\
\text { forcement. }\end{array}$ \\
\hline 16 & Potoski, M., Prakash, A. & 2005 & $\begin{array}{l}\text { Journal of Policy } \\
\text { Analysis and Manage- } \\
\text { ment }\end{array}$ & Empirical & Environmental performance; ISO 14001; Third party audits. \\
\hline 17 & Prakash, A., Potoski, M. & 2006 & $\begin{array}{l}\text { American Journal of } \\
\text { Political Science }\end{array}$ & Empirical & $\begin{array}{l}\text { Environmental governance; ISO 14001; Environmental regula- } \\
\text { tions. }\end{array}$ \\
\hline
\end{tabular}




\begin{tabular}{|c|c|c|c|c|c|}
\hline 18 & Tsai, W.-H., Chou, W.-C. & 2009 & $\begin{array}{l}\text { Expert Systems with } \\
\text { Applications }\end{array}$ & Empirical & $\begin{array}{l}\text { Analytic network process (ANP); Decision Making Trial and Eval- } \\
\text { uation Laboratory (DEMATEL); Management system; Sustainable } \\
\text { development; Zero-one goal programming (ZOGP). }\end{array}$ \\
\hline 19 & Rondinelli, D., Vastag, G. & 2000 & $\begin{array}{l}\text { European Manage- } \\
\text { ment Journal }\end{array}$ & Case study & $\begin{array}{l}\text { Certification; Environmental Management; ISO 14001; Quality } \\
\text { Management. }\end{array}$ \\
\hline 20 & $\begin{array}{l}\text { Heras-Saizarbitoria, I., Boiral, } \\
\text { O. }\end{array}$ & 2013 & $\begin{array}{l}\text { International Journal } \\
\text { of Management Re- } \\
\text { views }\end{array}$ & Review & $\begin{array}{l}\text { ISO 14001; ISO 9001; Management system standards; Meta-stand- } \\
\text { ards; Global governance. }\end{array}$ \\
\hline 21 & Ljungberg, L.Y. & 2007 & Materials and Design & Review & $\begin{array}{l}\text { Design; Ecology; Environmental impact; Materials selection; Re- } \\
\text { cycling; Sustainable product development. }\end{array}$ \\
\hline 22 & Robèrt, K.-H. & 2000 & $\begin{array}{l}\text { Journal of Cleaner } \\
\text { Production }\end{array}$ & Review & $\begin{array}{l}\text { Sustainable development; Sustainability; Systems thinking; Back- } \\
\text { casting; Strategies; Strategic planning; Ecological footprinting; } \\
\text { ISO 14001; EMAS; EMS; System conditions; The Natural Step } \\
\text { (TNS). }\end{array}$ \\
\hline 23 & Jiang, R.J., Bansal, P. & 2003 & $\begin{array}{l}\text { Journal of Manage- } \\
\text { ment Studies }\end{array}$ & $\begin{array}{l}\text { Empirical } \\
\text { (qualitative) }\end{array}$ & Environmental management systems; ISO 14001; Paper industry. \\
\hline 24 & Delmas, M. & 2001 & $\begin{array}{l}\text { Production and Opera- } \\
\text { tions Management }\end{array}$ & Empirical & $\begin{array}{l}\text { Competitive advantage; Natural environment; Organizational capa- } \\
\text { bility; Resource-based view; Stakeholders; Structural equation } \\
\text { modeling. }\end{array}$ \\
\hline 25 & $\begin{array}{l}\text { Govindan, K., Khodaverdi, R., } \\
\text { Vafadarnikjoo, A. }\end{array}$ & 2015 & $\begin{array}{l}\text { Expert Systems with } \\
\text { Applications }\end{array}$ & Case study & $\begin{array}{l}\text { DEMATEL; Green performances; Green practices; Green supply } \\
\text { chain management (GSCM); Intuitionistic fuzzy set (IFS). }\end{array}$ \\
\hline
\end{tabular}




\begin{tabular}{|c|c|c|c|c|c|}
\hline 26 & Kitazawa, S., Sarkis, J. & 2000 & $\begin{array}{l}\text { International Journal } \\
\text { of Operations and Pro- } \\
\text { duction Management }\end{array}$ & Case study & $\begin{array}{l}\text { Environmental management systems; International standards; Or- } \\
\text { ganizational centre; TQM. }\end{array}$ \\
\hline 27 & $\begin{array}{l}\text { Jørgensen, T.H., Remmen, A., } \\
\text { Mellado, M.D. }\end{array}$ & 2006 & $\begin{array}{l}\text { Journal of Cleaner } \\
\text { Production }\end{array}$ & Review & $\begin{array}{l}\text { Compatibility; Coordinated generic processes; Integrated manage- } \\
\text { ment systems; Integration; ISO 14001; ISO 9001; Management } \\
\text { system; OHSAS 18001; SA } 8000 .\end{array}$ \\
\hline 28 & Bansal, P. & 2002 & $\begin{array}{l}\text { Academy of Manage- } \\
\text { ment Executive }\end{array}$ & $\begin{array}{l}\text { Empirical } \\
\text { (qualitative) }\end{array}$ & $\begin{array}{l}\text { ISO 14001; Sustainable development; International en-vironment } \\
\text { systems; In-house environmental systems. }\end{array}$ \\
\hline 29 & Chan, E.S.W., Wong, S.C.K. & 2006 & Tourism Management & Empirical & $\begin{array}{l}\text { Discriminant analysis; EMS; Hotel industry; ISO 14001; Predictive } \\
\text { model. }\end{array}$ \\
\hline 30 & $\begin{array}{l}\text { Hsu, C.-C., Tan, K.C., Zailani, } \\
\text { S.H.M., Jayaraman, V. }\end{array}$ & 2013 & $\begin{array}{l}\text { International Journal } \\
\text { of Operations and Pro- } \\
\text { duction Management }\end{array}$ & Empirical & $\begin{array}{l}\text { Design for environment; Emerging economy; Green purchasing; } \\
\text { Green supply chain; Malaysia; Newly in-dustrialised economies; } \\
\text { Reverse logistics; Supply chain management; Sustainable develop- } \\
\text { ment. }\end{array}$ \\
\hline 31 & $\begin{array}{l}\text { González, P., Sarkis, J., } \\
\text { Adenso-Díaz, B. }\end{array}$ & 2008 & $\begin{array}{l}\text { International Journal } \\
\text { of Operations and Pro- } \\
\text { duction Management }\end{array}$ & Empirical & $\begin{array}{l}\text { Automotive industry; Environmental management; International } \\
\text { standards; Spain; Supply chain management. }\end{array}$ \\
\hline 32 & Zeng, S.X., Shi, J.J., Lou, G.X. & 2007 & $\begin{array}{l}\text { Journal of Cleaner } \\
\text { Production }\end{array}$ & Empirical & $\begin{array}{l}\text { Environment; Health and safety; Integrated management system; } \\
\text { ISO 14001; ISO 9001; OHSAS 18001; Quality; Synergy. }\end{array}$ \\
\hline 33 & $\begin{array}{l}\text { Nakamura, M., Takahashi, T., } \\
\text { Vertinsky, I. }\end{array}$ & 2001 & $\begin{array}{l}\text { Journal of Environ- } \\
\text { mental Economics and } \\
\text { Management }\end{array}$ & Empirical & $\begin{array}{l}\text { Decision making; Environmental economics; Industrial regulation; } \\
\text { Strategic approach. }\end{array}$ \\
\hline
\end{tabular}




\begin{tabular}{|c|c|c|c|c|c|}
\hline 34 & Bansal, P., Bogner, W.C. & 2002 & Long Range Planning & Empirical & $\begin{array}{l}\text { ISO 14001; Costs; Benefits; Environmental responsiveness; Insti- } \\
\text { tutions. }\end{array}$ \\
\hline 35 & $\begin{array}{l}\text { Laosirihongthong, T., } \\
\text { Adebanjo, D., Choon Tan, K. }\end{array}$ & 2013 & $\begin{array}{l}\text { Industrial Manage- } \\
\text { ment \& Data Systems }\end{array}$ & Empirical & $\begin{array}{l}\text { Performance; Institutional theory; Green supply chain manage- } \\
\text { ment. }\end{array}$ \\
\hline
\end{tabular}

Note: Sorted from highest to lowest number of citations. 
Additional analyses through this systematic review revealed that several researchers discussed about the rationale for using ISO 14001. The findings reported that there were 404 documents results when the keywords "ISO 14001"AND "Benefits" were included in the search query. On the other hand, there were 328 document results when "ISO 14001" AND "Costs" were used (according to the results from the systematic review - stage 1).

Furthermore, the systematic analysis (stage 2) sheds light on the latest contributions that were published in the last 7 years, specifically between January 2015 and December 2021. ISO published its third edition of ISO 14001 in October of the same year. During this period, there were 497 articles that were published in English, in peer reviewed journals. The review indicated that the top 10 subject areas were related to Business, Management and Accounting (250); Environmental Science (244); Engineering (138); Social Sciences (127); Energy (117); Economics, Econometrics and Finance (55); Decision Sciences (50); Computer Science (28); Medicine (22) and Chemical Engineering (18).

Again, with 57 publications, the Journal of Cleaner Production was ranked as the top journal. Other outlets included Sustainability Switzerland (28); Business Strategy and the Environment (23); Quality Access to Success (12); Corporate Social Responsibility and Environmental Management (10); Environmental Engineering and Management Journal (9); Total Quality Management and Business Excellence (9); International Journal for Quality Research (8); TQM Journal (8); and Environmental Quality Management (7). Table 3 presents a list of 35 most-cited articles between January 2015 and December 2021. To date, these contributions accumulated more than 50 citations in Scopus. 
Table 3. List of most-cited academic articles on ISO 14001 - between 2015 and 2021

\begin{tabular}{|c|c|c|c|c|c|}
\hline $\begin{array}{c}\text { Scopus } \\
\text { Rank }\end{array}$ & Authors & Year & Source title & Research Type & Keywords \\
\hline $1^{*}$ & $\begin{array}{l}\text { Govindan, K., Khodaverdi, } \\
\text { R., Vafadarnikjoo A. }\end{array}$ & 2015 & $\begin{array}{l}\text { Expert Systems } \\
\text { with Applications }\end{array}$ & Empirical & $\begin{array}{l}\text { DEMATEL; Green performances; Green practices; Green } \\
\text { supply chain management (GSCM); Intuitionistic fuzzy set } \\
\text { (IFS). }\end{array}$ \\
\hline 2 & $\begin{array}{l}\text { Abdul-Rashid, S.H., Sa- } \\
\text { kundarini, N., Raja } \\
\text { Ghazilla, R.A., } \\
\text { Thurasamy, R. }\end{array}$ & 2017 & $\begin{array}{l}\text { International Jour- } \\
\text { nal of Operations } \\
\text { and Production } \\
\text { Management }\end{array}$ & Empirical & $\begin{array}{l}\text { Economic performance; Environmental performance; Social } \\
\text { performance; Structural equation modelling; Sustainability } \\
\text { performance; Sustainable manufacturing practices. }\end{array}$ \\
\hline 3 & $\begin{array}{l}\text { Teixeira, A.A., Jabbour, } \\
\text { C.J.C., De Sousa Jabbour, } \\
\text { A.B.L., Latan, H., De } \\
\text { Oliveira, J.H.C. }\end{array}$ & 2016 & $\begin{array}{l}\text { Journal of Cleaner } \\
\text { Production }\end{array}$ & Empirical & $\begin{array}{l}\text { Brazil; Green human resource management; Green supply } \\
\text { chain; Green training; Sustainable management; Sustainable } \\
\text { operations. }\end{array}$ \\
\hline 4 & $\begin{array}{l}\text { Miroshnychenko, I., } \\
\text { Barontini, R., Testa, F. }\end{array}$ & 2017 & $\begin{array}{l}\text { Journal of Cleaner } \\
\text { Production }\end{array}$ & Empirical & $\begin{array}{l}\text { Financial performance; Green product; Green supply chain } \\
\text { management; ISO 14001; Pollution prevention. }\end{array}$ \\
\hline 5 & $\begin{array}{l}\text { Testa, F., Boiral, O., } \\
\text { Iraldo, F. }\end{array}$ & 2018 & $\begin{array}{l}\text { Journal of Business } \\
\text { Ethics }\end{array}$ & Empirical & $\begin{array}{l}\text { Environmental management system; Environmental strat- } \\
\text { egy; Greenwashing; Institutional complexity; Stakeholders. }\end{array}$ \\
\hline 6 & $\begin{array}{l}\text { Latan, H., Chiappetta Jab- } \\
\text { bour, C.J., Lopes de Sousa } \\
\text { Jabbour, A.B., Wamba, } \\
\text { S.F., Shahbaz, M. }\end{array}$ & 2018 & $\begin{array}{l}\text { Journal of Cleaner } \\
\text { Production }\end{array}$ & Empirical & $\begin{array}{l}\text { Corporate environmental performance; Environmental man- } \\
\text { agement accounting; Environmental strategy; Perceived en- } \\
\text { vironmental uncertainty; Top Management's commitment. }\end{array}$ \\
\hline 7 & $\begin{array}{l}\text { Bernardo, M., Simon, A., } \\
\text { Tarí, J.J., Molina-Azorín, } \\
\text { J.F. }\end{array}$ & 2015 & $\begin{array}{l}\text { Journal of Cleaner } \\
\text { Production }\end{array}$ & Review & $\begin{array}{l}\text { Benefits; Integrated management sys- } \\
\text { tem; ISO 14001; ISO 9001; Literature review; Performance. }\end{array}$ \\
\hline
\end{tabular}


Boiral, O., Guillaumie, L.,

8

Heras-Saizarbitoria, I.,

Tayo Tene, C.V.

\begin{tabular}{|c|c|c|c|c|c|}
\hline & Tayo Tene, C.V. & & Reviews & & \\
\hline 9 & Jabbour, C.J.C. & 2015 & $\begin{array}{l}\text { Journal of Cleaner } \\
\text { Production }\end{array}$ & Empirical & $\begin{array}{l}\text { Brazil; Environmental management; Environmental train- } \\
\text { ing; Green human resource management; ISO 14001; Sus- } \\
\text { tainability. }\end{array}$ \\
\hline 10 & $\begin{array}{l}\text { Abu Seman, N.A., Go- } \\
\text { vindan, K., Mardani, A., } \\
\text { Zakuan, N., Mat Saman, } \\
\text { M.Z., Hooker, R.E., } \\
\text { Ozkul, S. }\end{array}$ & 2019 & $\begin{array}{l}\text { Journal of Cleaner } \\
\text { Production }\end{array}$ & Empirical & $\begin{array}{l}\text { Environmental; Environmental performance; Green innova- } \\
\text { tion; Green supply chain management (GSCM); Manufac- } \\
\text { turing industry. }\end{array}$ \\
\hline 11 & Souza, J.P.E., Alves, J.M. & 2018 & $\begin{array}{l}\text { Journal of Cleaner } \\
\text { Production }\end{array}$ & Empirical & $\begin{array}{l}\text { Corporate sustainability; ISO 14001; ISO 26000; ISO 9001; } \\
\text { Lean manufacturing; OHSAS 18001. }\end{array}$ \\
\hline 12 & Scur, G., Barbosa, M.E. & 2017 & $\begin{array}{l}\text { Journal of Cleaner } \\
\text { Production }\end{array}$ & Case Study & $\begin{array}{l}\text { Electrical-electronic industry; Green practices; Green sup- } \\
\text { ply chain management; Home appliance. }\end{array}$ \\
\hline 13 & $\begin{array}{l}\text { Martín-de Castro, G., } \\
\text { Amores-Salvadó, J., Na- } \\
\text { vas-López, J.E. }\end{array}$ & 2016 & $\begin{array}{l}\text { Corporate Social } \\
\text { Responsibility and } \\
\text { Environmental } \\
\text { Management }\end{array}$ & Empirical & $\begin{array}{l}\text { Environmental management systems; Environmental pol- } \\
\text { icy; Firm performance; Green corporate im- } \\
\text { age; ISO 14001; Natural resource-based view; Stakeholder } \\
\text { engagement. }\end{array}$ \\
\hline 14 & $\begin{array}{l}\text { Husted, B.W., Montiel, I., } \\
\text { Christmann, P. }\end{array}$ & 2016 & $\begin{array}{l}\text { Journal of Interna- } \\
\text { tional Business } \\
\text { Studies }\end{array}$ & Empirical & $\begin{array}{l}\text { Corporate social responsibility; Emerging markets/coun- } \\
\text { tries/economies; Geographic distance; Liability of foreign- } \\
\text { ness; Location strategy. }\end{array}$ \\
\hline 15 & $\begin{array}{l}\text { Campos, L.M.S., De Melo } \\
\text { Heizen, D.A., Verdinelli, } \\
\text { M.A., Cauchick Miguel, } \\
\text { P.A. }\end{array}$ & 2015 & $\begin{array}{l}\text { Journal of Cleaner } \\
\text { Production }\end{array}$ & Empirical & $\begin{array}{l}\text { EMS; Environmental management system; Environmental } \\
\text { performance indicators; ISO 14001; Survey research. }\end{array}$ \\
\hline 16 & Nguyen, Q.A., Hens, L. & 2015 & $\begin{array}{l}\text { Journal of Cleaner } \\
\text { Production }\end{array}$ & Empirical & $\begin{array}{l}\text { Cement; Environmental management systems; Environmen- } \\
\text { tal performance; ISO 14001; Vietnam. }\end{array}$ \\
\hline
\end{tabular}

ISO14001 impacts; inclusion criteria; exclusion criteria; ISO14001 outcomes; ISO14001 adoption.

International Jour-

nal of Management Review

Hooker, R.E., 


\begin{tabular}{|c|c|c|c|c|c|}
\hline 17 & $\begin{array}{l}\text { Lee, V.-H., Ooi, K.-B., } \\
\text { Chong, A.Y.-L., Lin, B. }\end{array}$ & 2015 & $\begin{array}{l}\text { Production Plan- } \\
\text { ning and Control }\end{array}$ & Empirical & $\begin{array}{l}\text { Competitive advantage; Environmental performance; Green- } \\
\text { ing the supplier; Malaysia. }\end{array}$ \\
\hline 18 & Zorpas, A.A. & 2020 & $\begin{array}{l}\text { Science of the To- } \\
\text { tal Environment }\end{array}$ & Review & $\begin{array}{l}\text { Circular economy; European Green Deal; LCA; PESTEL } \\
\text { analysis; Prevention strategy; Strategy development; SWOT } \\
\text { analysis; Waste strategy; Zero waste approach. }\end{array}$ \\
\hline 19 & $\begin{array}{l}\text { Heras-Saizarbitoria, I., } \\
\text { Arana, G., Boiral, O. }\end{array}$ & 2016 & $\begin{array}{l}\text { Business Strategy } \\
\text { and the Environ- } \\
\text { ment }\end{array}$ & Empirical & $\begin{array}{l}\text { EMAS; environmental management; environmental man- } \\
\text { agement systems; ISO 14001; Spain. }\end{array}$ \\
\hline 20 & $\begin{array}{l}\text { Arimura, T.H., Darnall, N., } \\
\text { Ganguli, R., Katayama, H. }\end{array}$ & 2016 & $\begin{array}{l}\text { Journal of Environ- } \\
\text { mental Manage- } \\
\text { ment }\end{array}$ & Empirical & $\begin{array}{l}\text { Certification program; Discrete choice model; Endogene- } \\
\text { ity; Environmental performance; ISO 14001, environmental } \\
\text { management system; Voluntary environmental program. }\end{array}$ \\
\hline 21 & $\begin{array}{l}\text { Vijayvargy, L., Thakkar, } \\
\text { J., Agarwal, G. }\end{array}$ & 2017 & $\begin{array}{l}\text { Journal of Manu- } \\
\text { facturing Technol- } \\
\text { ogy Management }\end{array}$ & Empirical & $\begin{array}{l}\text { Emerging economies; Empirical study; Green supply chain } \\
\text { management; Organizational size. }\end{array}$ \\
\hline 22 & $\begin{array}{l}\text { Oliveira, J.A., Oliveira, } \\
\text { O.J., Ometto, A.R., Fer- } \\
\text { raudo, A.S., Salgado, M.H. }\end{array}$ & 2016 & $\begin{array}{l}\text { Journal of Cleaner } \\
\text { Production }\end{array}$ & Empirical & $\begin{array}{l}\text { Cleaner production; Environmental management system; In- } \\
\text { dustrial companies; ISO } 14001 .\end{array}$ \\
\hline 23 & $\begin{array}{l}\text { Su, H.-C., Dhanorkar, S., } \\
\text { Linderman, K. }\end{array}$ & 2015 & $\begin{array}{l}\text { Journal of Opera- } \\
\text { tions Management }\end{array}$ & Empirical & $\begin{array}{l}\text { Absorptive capacity; Competitive strategy; Early mover ad- } \\
\text { vantage; ISO 14001; ISO 9001; Management standards. }\end{array}$ \\
\hline 24 & $\begin{array}{l}\text { Ikram, M., Zhang, Q., } \\
\text { Sroufe, R., Shah, S.Z.A. }\end{array}$ & 2020 & $\begin{array}{l}\text { Sustainable Pro- } \\
\text { duction and Con- } \\
\text { sumption }\end{array}$ & Empirical & $\begin{array}{l}\text { CO2; Emission; GRA; Grey TOPSIS; ISO 14001; Renewa- } \\
\text { ble energy; Sustainability. }\end{array}$ \\
\hline 25 & Iatridis, K., Kesidou, E. & 2018 & $\begin{array}{l}\text { Journal of Business } \\
\text { Ethics }\end{array}$ & $\begin{array}{l}\text { Empirical (quali- } \\
\text { tative) }\end{array}$ & $\begin{array}{l}\text { Economic crisis; Environmental management; Greece; Inter- } \\
\text { nalization; ISO 14001; Motivations. }\end{array}$ \\
\hline 26 & $\begin{array}{l}\text { Nunhes, T.V., Ferreira } \\
\text { Motta, L.C., de Oliveira, } \\
\text { O.J. }\end{array}$ & 2016 & $\begin{array}{l}\text { Journal of Cleaner } \\
\text { Production }\end{array}$ & Review & $\begin{array}{l}\text { Integrated management system; ISO 14001; ISO 9001; Man- } \\
\text { agement systems; OHSAS } 18001 .\end{array}$ \\
\hline
\end{tabular}


Shaharudin, M.R., Go-

27 vindan, K., Zailani,
S., Tan, K.C., Iranmanesh,
Production Empirical

2017 Journal of Cleaner

Activities; Closed-loop supply chains; Effectiveness; ProdM.

\begin{tabular}{|c|c|c|c|c|c|}
\hline 28 & $\begin{array}{l}\text { Singh, M., Brueckner, M., } \\
\text { Padhy, P.K. }\end{array}$ & 2015 & $\begin{array}{l}\text { Journal of Cleaner } \\
\text { Production }\end{array}$ & Empirical & $\begin{array}{l}\text { Environmental management systems; Environmental perfor- } \\
\text { mance; ISO 140001; Policies; Small and medium-sized en- } \\
\text { terprises; Waste minimisation. }\end{array}$ \\
\hline 29 & $\begin{array}{l}\text { Ikram, M., Mahmoudi, A., } \\
\text { Shah, S.Z.A., Mohsin, M. }\end{array}$ & 2019 & $\begin{array}{l}\text { Environmental Sci- } \\
\text { ence and Pollution } \\
\text { Research }\end{array}$ & Empirical & $\begin{array}{l}\text { Competitive advantage; Doubling time; Environmental sus- } \\
\text { tainability; Forecasting; ISO 14001; Relative growth rate }\end{array}$ \\
\hline 30 & da Fonseca, L.M.C.M. & 2015 & $\begin{array}{l}\text { Journal of Indus- } \\
\text { trial Engineering } \\
\text { and Management }\end{array}$ & Review & $\begin{array}{l}\text { Environmental management systems; ISO } 14001 \text { revision; } \\
\text { Standardization; Sustainability. }\end{array}$ \\
\hline 31 & $\begin{array}{l}\text { Jabbour, C.J.C, Seuring, } \\
\text { S., Lopes de Sousa Jab- } \\
\text { bour, A.B., (...), Latan, H., } \\
\text { Izeppi, W.C. }\end{array}$ & 2020 & $\begin{array}{l}\text { Journal of Environ- } \\
\text { mental Manage- } \\
\text { ment }\end{array}$ & Empirical & $\begin{array}{l}\text { Circular economy; Stakeholders; Sustainable business mod- } \\
\text { els; Sustainable innovation; Sustainable performance. }\end{array}$ \\
\hline 32 & Ahmed, W., Najmi, A. & 2018 & $\begin{array}{l}\text { Management of } \\
\text { Environmental } \\
\text { Quality: An Inter- } \\
\text { national Journal }\end{array}$ & Empirical & $\begin{array}{l}\text { External green collaboration; Green supply chain manage- } \\
\text { ment; Institutional pressures; Internal green practices; Struc- } \\
\text { tural equation modelling. }\end{array}$ \\
\hline 33 & $\begin{array}{l}\text { Hojnik, J., Ruzzier, M., } \\
\text { Manolova, T.S. }\end{array}$ & 2018 & $\begin{array}{l}\text { Journal of Cleaner } \\
\text { Production }\end{array}$ & Empirical & $\begin{array}{l}\text { Eco-innovation; Firm performance; Internationaliza- } \\
\text { tion; ISO14001; Mediating effect; Sustainability. }\end{array}$ \\
\hline 34 & $\begin{array}{l}\text { Xu, X.D., Zeng, S.X., Zou, } \\
\text { H.L., Shi, J.J. }\end{array}$ & 2016 & $\begin{array}{l}\text { Business Strategy } \\
\text { and the Environ- } \\
\text { ment }\end{array}$ & Empirical & $\begin{array}{l}\text { Environmental violation events; Event study; Media cover- } \\
\text { age; Shareholders' wealth. }\end{array}$ \\
\hline
\end{tabular}


Salim, H.K., Padfield, R.,

35 Hansen, S.B., (...), Tham,

M.H., Papargyropoulou, E.
2018 Journal of Cleaner

Production
Bibliometric analysis; Environmental management system;

ISO 14001; Multi-stakeholder framework; Thematic trends.

Note: Sorted from highest to lowest number of citations.

*Govindan et al. (2015) was also reported in Table 2 
In the past seven years, the most used keywords included: ISO 14001 (224); Environmental Management (150); Environmental Management Systems (98); Sustainable Development (66); Sustainability (64); Environmental Management System (59); Environmental Performance (57); Certification (51); ISO 9001 (42) and EMAS (28).

This extended list indicated that there were much fewer contributions whose keywords were focused on society's contentious sustainability issues including climate change (9), pollution (7), carbon footprint (6) and greenhouse gas emissions (5), among other topics.

Furthermore, the search results (between 2015-2021) indicated that there were 95 documents results when the keywords "ISO "14001" AND "Benefits", and just 56 documents were retrieved when "ISO 14001" AND "Costs" were inserted in the query.

\section{A benefit-cost analysis of ISO 14001}

\subsection{Benefits}

The findings revealed that practitioners developed, implemented, managed, coordinated and monitored their environmental activities to improve their competitive position/competitive advantage (Ikram et al., 2019; Hojnik et al., 2018; Oliveira et al., 2016;) and profitability (Delmas, 2002). Standards like ISO 14001 are meant to support organizations in the development of environmental objectives, delegation of responsibilities, the provision of training and development, as well as in formulating performance management audits (Santos, Rebelo, Lopes, Alves \& Silva, 2016; Bernardo et al., 2015; Da Fonseca, 2015; Delmas, 2002; Hojnik et al., 2018; Hsu et al., 2013; Nunhes et al., 2016; Oliveira et al., 2016; Su et al., 2015). ISO has created a system whereby third-party auditors can certify the organizations' conformity with relevant environmental standards. Practitioners can use ISO 14001 as a framework to evaluate 
their environmental practices and externalities. They can compare them across various industries and jurisdictions (Boiral et al., 2018).

4.1.1 ISO 14001 raises awareness on compliance requirements with relevant legislation

ISO 14001 can help organizations to embed environmental management issues in their corporate strategies and in their day-to-day operations. Businesses can use its substantive environment management systems to adopt beyond-compliance measures. They could even anticipate their jurisdiction's regulatory requirements (Boiral et al., 2018; Husted et al., 2016) (to confirm that they are reducing their pollution levels for the purpose of avoiding sanctions). They may follow ISO 14001 guidelines to better identify certain aspects of their organization's activities to determine their significance, priorities and impact on the environment.

4.1.2 ISO 14001 can facilitate planning, organization, leadership and control of environmental management systems

Practitioners can establish a program to implement environment management policies (with a disciplined process of evaluating and achieving target performance levels) while seeking continuous improvements (Camilleri, 2019b, Delmas, 2001; Santos, et al., 2016; Shaharudin et al., 2017; Vijayvargy et al., 2017). They are bound to increase the involvement of their managers and have to delegate employees with a clear assignment of duties, to pursue responsible environmental behaviors. Organizational leaders should allocate relevant resources and foster facilitating conditions to implement ISO 14001, including the provision of ongoing training and development to employees, to achieve the standards' underlying objectives, including increasing operational efficiencies and cost savings (Camilleri, 2021b). They are expected to regularly review and audit their environment management systems and to identify opportunities for improvement, in terms of environmental performance. 
4.1.3 ISO 14001 can be used to monitor and reduce externalities including pollution and emissions

The standard's certification ought to confirm that practitioners are implementing responsible practices to improve their facilities' environmental performance, by reducing their pollution emissions (Garrido, González \& Orcos, 2020; Ikram et al., 2020; Oliveira et al., 2016; Potoski \& Prakash, 2005a, 2005b, Prakash \& Potoski, 2006). ISO 14001 clearly identifies the operational requirements that have to be taken on board by companies to minimize their environmental impact, by reducing pollution and emissions (Su et al., 2015).

4.1.4 ISO 14001 can be used to establish and maintain ongoing communications with stakeholders

Practitioners are expected to utilize ISO 14001 to raise awareness about their environment management systems with interested parties. Their corporate communications can help them alleviate their constituents' concerns about environmental performance. Like other standards, the development of ISO 14001 facilitates exchanges and communications among a wide array of stakeholders (Da Fonseca, 2015; Nunhes et al., 2016; Salim et al., 2018; Su et al., 2015).

\subsubsection{ISO 14001 can used to increase the legitimacy of practitioners in society}

The adoption of environmental management practices can improve corporate reputation and may enhance the practitioners' transparency credentials with stakeholders (Da Fonseca, 2015; Oliveira et al., 2016; Su, et al., 2015). Firms can manage their legitimacy by conveying (material) information regarding operational improvements in their processes and by clearly demonstrating their ongoing commitment to the environment (Camilleri, 2021b; Mtapuri, Camilleri \& Dłużewska, 2021; Santos et al., 2016). 
4.1.6 ISO 14001 can create shared value to corporate financial performance and to the environment

Previous research indicated that stakeholders recognized (and awarded) practitioners for their environmental performance credentials. Very often, responsible corporate environmental practices led to a positive financial performance (Martín-de Castro et al., 2017) and to a significant increase in the companies' stock market values (Shaharudin et al., 2017; Su et al., 2018). Eco-efficient companies are increasingly rewarding their shareholders with a greater return on investment (Melnyk et al., 2003). Notwithstanding, investing firms are recommending positive impact, financial portfolios that are based on the firms' environmental records (Camilleri, 2021c; Su et al., 2018).

Green supply chain initiatives have a direct impact on the firms' performance outcomes as well as on their external environment (Miroshnychenko et al., 2017; Vijayvargy et al., 2017). Businesses can safeguard the natural environment by reducing the utilization of raw materials, by minimizing waste from manufacturing processes, and by reusing "waste" resources when designing products (Camilleri, 2019a, 2020, Scur \& Barbosa, 2017; Delmas, 2002; Shaharudin, et al., 2017; Souza \& Alves, 2018; Vijayvargy et al., 2017). Such responsible behaviors can also translate to economic benefits, operational efficiencies and less costs for the producers of goods (Curkovic \& Sroufe, 2011; Govindan et al., 2015; Iatridis \& Kesidou, 2018; Laosirihongthong et al., 2013; Scur et al., 2017; Souza \& Alves, 2018; Teixeira et al., 2016). Previous research confirmed that the businesses' credentials in corporate environmental performance can improve the value of their firm (Kong et al., 2015) and could be in a better position to attract new shareholders (Angelia \& Suryaningsih, 2015; Heras-Saizarbitoria et al., 2016). 


\subsection{Costs}

4.2.1 ISO 14001 requires significant investments in time and money

Prospective practitioners ought to consider the opportunity costs before implementing ISO 14001. They have to ascertain whether it would be profitable for their company to invest resources, in terms of time and money, before committing themselves to certify their facilities (Delmas \& Toffel, 2008). Failure to comply can result in fines (in some jurisdictions) for not meeting the environmental standards' criteria, as well as in increased interventions from the part of regulators (Melnyk et al., 2003). The certification of ISO 14001 would necessitate that organizations pay an initiation fee as well as ongoing membership dues. They are expected to adhere to the requirements of the substantive standard and for annual recertification audits (Potoski \& Prakash, 2005).

\subsubsection{Lack of information on the difference between certified and uncertified ISO 14001}

Little is known about how outsiders interpret the certification of voluntary standards (Arimura et al., 2016; Camilleri, 2019b; Husted et al., 2016). Although ISO 14001 maps out an assurance mechanism for effective environment management systems; it does not clarify the exact requirements (in detail) on how to obtain its certification. ISO clearly specifies that this generic standard can be used by any organization (in any industry) that wants to improve its resource efficiency and cost savings, minimize waste, emissions and pollution to the environment (Eltayeb et al., 2011; Hsu et al., 2013; Melnyk et al., 2003; Morrow \& Rondinelli, 2002; Nunhes et al., 2016; Shaharudin, et al., 2017).

ISO 14001 does not constrain the operations of practitioners although they may be pressurized by institutions and by other stakeholders to adopt certified management standards. Therefore, businesses are encouraged to certify their premises to conform to acceptable 
environmental practices. Such certification would enable them to prove their social license to operate (Demuijnck \& Fasterling, 2016; Hsu et al., 2013; Soewarno et al., 2019; Wilburn \& Wilburn, 2011).

4.2.3 Stakeholders exert their pressures on corporate businesses and organizations to adopt environmental management practices

Organizations may be intrigued to adopt ISO 14001 to appease different stakeholders including governments, activists, local communities, trade associations, investors, and customers, each of which possess their own culture, interests and conception of legitimate environmental management practices (Ahmed \& Najmi, 2018; Bansal \& Bogner, 2002; Colwell \& Joshi, 2013; Delmas \& Toffel, 2008). Previous research indicated that many companies participate in such environment management programs to forge closer relationships with the government and its agencies (Clemens \& Douglas, 2005; Delmas, 2002; Jabbour et al., 2020; Morrow \& Rondinelli, 2002).

Practitioners may experience institutional pressures during periods of uncertainty, before formalizing their organizations' environmental practices in corporate social responsibility reports (or when they are preparing their integrated disclosures of financial and non-financial performance) that they may have to submit to regulatory authorities (Ntim \& Soobaroyen, 2013; Testa et al., 2018).

4.2.4 ISO 14001 necessitates continuous commitment from the part of management and employees

Organizational leaders are expected to communicate with management and employees about their environmental management compliance objectives and strategies (Testa et al., 2018; Santos et al., 2016; Zorpas, 2020). They have to clarify their courses of action before applying 
for ISO 14001 certification, whilst they are implementing it, and when they are measuring and controlling its effectiveness (Latan et al., 2018). Hence, they should organize training and development opportunities for their members of staff, to instill their motivation to pursue cultural changes, and to foster continuous improvements in environmentally responsible behaviors (Boiral, 2007; Colwell \& Joshi, 2013; Heras-Saizarbitoria \& Boiral, 2013; Jabbour, 2015). Employees ought to be incentivized to contribute to the achievement of their organizations' environmental performance targets (Abdul-Rashid et al., 2017; Agan et al., 2016; Darnall, 2006; Jacobs et al., 2010; Ljungberg, 2007; Potoski \& Prakash, 2005; Rennings et al., 2006; Russo, 2009; Testa et al., 2014; Zailani et al., 2012).

\subsubsection{Lack of consensus on the actual effectiveness of ISO 14001}

Various academic commentators clearly pointed out that there are different shades of opinion on the effectiveness of ISO 14001 (Boiral et al., 2018; Iatridis \& Kesidou, 2018; Testa, et al., 2018; Zorpas, 2020). This environment management standard comprises a set of principles and guidelines that may be perceived as a 'rational myth' that spurs the organizations' 'ceremonial behaviors' (Nunhes et al., 2016). Many researchers argued that although ISO 14001 provides a certification from an independent body about the existence of an environmental management system, this voluntary instrument does not necessarily translate to superior performance (Arimura, Darnall \& Katayama, 2011; Boiral et al., 2018; Prakash \& Potoski, 2006). Under these circumstances, ISO's norms may not always play a significant role in promoting environmentally responsible behaviors, unless businesses are compelled by regulatory authorities to adopt ISO 14001's standardized practices and to report about their performance in corporate non-financial disclosures (Camilleri, 2015b; EU 2021). 


\section{Conclusions and implications}

This research reported that ISO 14001's environment management systems can be employed by small, medium or large businesses operating in various industry sectors, in different contexts. A thorough literature review revealed that the adoption of ISO 14001 may be triggered by institutions and stakeholders. The governments and their regulatory authorities can exert their pressures on organizations to follow their rules, norms and ethical principles.

The practitioners' compliance with legislation, regulatory guidelines and principles can increase their legitimacy among stakeholders and could validate their social license to operate. Hence, there is scope for businesses and other organizations to be proactive by anticipating legislation. This way, they can achieve a competitive advantage by proving their environmental credentials with stakeholders.

The systematic review suggested that compliance with ISO 14001 may result in a number of benefits to practitioners like improving their facilities' environmental performance through resource efficiencies. At the same time, they could minimize their externalities to the environment. This research confirmed that such certifiable standards, can create synergistic value to the businesses' financial performance and to the environment. It reported that green supply chain initiatives as well as a better utilization of resources and waste management practices may translate to lower costs and to an improved financial performance.

In many cases, several authors clarified that practitioners could enhance their environmental management through waste reduction, circular economy approaches, increased productivities (cost savings, improved systemization practices and management control, improved employee results, reduction in mistakes and rework, shorter lead times) and the like. Other research reported that the practitioners' environmental credentials resulted in an improved corporate image among external stakeholders including customers, suppliers and regulatory authorities. 
Notwithstanding, this contribution suggested that it is in the interest of practitioners to communicate about their adoption of prescriptive environmental management systems and to shed light on their environmental performance. Their corporate environmental disclosures can boost their reputation, increase their trustworthiness and credibility among stakeholders, particularly if they promote their environmentally responsible behaviors through different media whilst forging relationships with different stakeholders.

On the other hand, various researchers noted that the main obstacles to ISO 14001 certification are related to high certification costs, superficial adoption, an increase in paperwork and red tape, time constraints, as well as the employees' resistance to change, and/or their lack of capabilities to comply with the standard's requirements, among other contentious issues. Very often, they argued that the implementation of ISO 14001 would necessitate an ongoing support and commitment from the part of managers as well as the involvement of employees, to integrate the standard's environment management systems with existing practices.

Organizational leaders who are considering the introduction of such environment systems are expected to communicate about their strategic plans with their management and members of staff. They have to motivate and incentivize each employee to achieve their corporate objectives and environmental performance targets.

\section{Limitations and future research directions}

Previous studies examined the stakeholders' perceptions about different aspects of ISO 14001, including on the implementation of ISO 14001, internal management support, integrated management systems, supply chain management, et cetera. Very often, their data was collected from employees and managers of certified entities.

This systematic review appraises previous articles that were focused on environment management systems. It reported some of the most cited papers since 1995, where it identified the 
authors, the publication outlets and the type of methodology that was used to capture the data. In addition, it indicated the keywords that were related to the articles' topics. Furthermore, this contribution provided a synthesis of the findings as it clarified the benefits and costs of using ISO 14001's environment management systems.

This contribution implies that for the time being, just a few contributions explored how, where and when organizations were using ISO14001 to address the latest global challenges including the effects of waste and pollution on climate change. For example, the systematic analyses revealed that few articles sought to explore how ISO 14001 could be used to minimize the carbon footprint and/or greenhouse gas emissions.

This paper calls for further empirical studies that rely on quantitative and/or qualitative approaches as well as discursive and/or theoretical papers on environment management systems and their related topics. Prospective research can utilize different research methods, sampling frames and analytical techniques to investigate the impacts of certified organizations and the efficacy of their performance indicators in different contexts. There is scope to shed more light on the companies' environmental credentials, particularly those operating in growing economies like China. A recent ISO survey indicated that China represented $48.25 \%$ (almost half) of the total ISO 14001 certifications that were issued in 2020 (ISO, 2021). Perhaps, longitudinal studies may be used to provide a better understanding of the long-term effects of ISO 14001 on the organizations' economic and operational performance in different industry sectors.

Acknowledgements: The author thanks the editor as well as the four reviewers for their constructive remarks and suggestions.

Conflicts of Interest: The author declares no conflict of interest. 


\section{References}

Abdul-Rashid, S. H., Sakundarini, N., Ghazilla, R. A. R., \& Thurasamy, R. (2017). The impact of sustainable manufacturing practices on sustainability performance: Empirical evidence from Malaysia. International Journal of Operations \& Production Management., 37(2), 182-204.

Agan, Y., Acar, M. F., \& Borodin, A. (2013). Drivers of environmental processes and their impact on performance: a study of Turkish SMEs. Journal of Cleaner Production, 51, 23-33.

Ahmed, W., \& Najmi, A. (2018). Developing and analyzing framework for understanding the effects of GSCM on green and economic performance: perspective of a developing country. Management of Environmental Quality: An International Journal, 29(4), 740-758.

Angelia, D., \& Suryaningsih, R. (2015). The effect of environmental performance and corporate social responsibility disclosure towards financial performance (Case study to manufacture, infrastructure, and service companies that listed at Indonesia stock exchange). Procedia-Social and Behavioral Sciences, 211, 348-355.

Arimura, T. H., Darnall, N., \& Katayama, H. (2011). Is ISO 14001 a gateway to more advanced voluntary action? The case of green supply chain management. Journal of Environmental Economics and Management, 61(2), 170-182.

Arimura, T. H., Darnall, N., Ganguli, R., \& Katayama, H. (2016). The Effect of ISO 14001 on Environmental Performance: Resolving Equivocal Findings, Journal of Environmental Management, 166, 556-566.

Baek, K. (2018). Sustainable development and pollutant outcomes: The case of ISO 14001 in Korea. Corporate Social Responsibility and Environmental Management, 25(5), 825-832.

Balluchi, F., Lazzini, A., \& Torelli, R. (2021). Credibility of environmental issues in non-financial mandatory disclosure: Measurement and determinants. Journal of Cleaner Production, $288,125744$.

Bansal, P., \& Bogner, W. C. (2002). Deciding on ISO 14001: economics, institutions, and context. Long Range Planning, 35(3), 269-290.

Beck, C., Dumay, J., \& Frost, G. (2017). In pursuit of a 'single source of truth': from threatened legitimacy to integrated reporting. Journal of Business Ethics, 141(1), 191-205.

Bernardo, M., Simon, A., Tarí, J. J., \& Molina-Azorín, J. F. (2015). Benefits of management systems integration: a literature review. Journal of Cleaner Production, 94, 260-267.

Benz, L., Paulus, S., Scherer, J., Syryca, J., \& Trück, S. (2021). Investors' carbon risk exposure and their potential for shareholder engagement. Business Strategy and the Environment, 30(1), 282-301.

Birindelli, G., \& Chiappini, H. (2021). Climate change policies: Good news or bad news for firms in the European Union?. Corporate Social Responsibility and Environmental Management, 28(2), 831-848. 
Boiral, O. (2007). Corporate greening through ISO 14001: a rational myth?. Organization Science, 18(1), 127-146.

Boiral, O., Guillaumie, L., Heras-Saizarbitoria, I., \& Tayo Tene, C. V. (2018). Adoption and outcomes of ISO 14001: A systematic review. International Journal of Management Reviews, 20(2), 411-432.

Boström, M., \& Hallström, K. T. (2010). NGO power in global social and environmental standard-setting. Global Environmental Politics, 10(4), 36-59.

Bravi, L., Santos, G., Pagano, A., \& Murmura, F. (2020). Environmental management system according to ISO 14001: 2015 as a driver to sustainable development. Corporate Social Responsibility and Environmental Management, 27(6), 2599-2614.

Camilleri, M. A. (2015a). Valuing stakeholder engagement and sustainability reporting. Corporate Reputation Review, 18(3), 210-222. https://doi.org/10.1057/crr.2015.9

Camilleri, M.A. (2015b). Environmental, social and governance disclosures in Europe. Sustainability Accounting, Management and Policy Journal, 6(2), 224-242. https://doi.org/10.1108/SAMPJ-10-2014-0065

Camilleri, M. A. (2016). Responsible tourism that creates shared value among stakeholders. Tourism Planning \& Development, 13(2), 219-235.

https://doi.org/10.1080/21568316.2015.1074100

Camilleri, M. A. (2017a). Corporate citizenship and social responsibility policies in the United States of America. Sustainability Accounting, Management and Policy Journal, 8(1), 77-93. https://doi.org/10.1108/SAMPJ-05-2016-0023

Camilleri, M. A. (2017b). The integrated reporting of financial, social and sustainability capitals: a critical review and appraisal. International Journal of Sustainable Society, 9(4), 311326. https://www.inderscienceonline.com/doi/abs/10.1504/IJSSOC.2017.090523

Camilleri, M.A. (2018). Theoretical insights on integrated reporting: The inclusion of nonfinancial capitals in corporate disclosures, Corporate Communications: An International Journal, 23(4), 567-581. https://doi.org/10.1108/CCIJ-01-2018-0016

Camilleri, M. A. (2019). Measuring the corporate managers' attitudes towards ISO's social responsibility standard. Total Quality Management \& Business Excellence, 30(13-14), 15491561. https://doi.org/10.1080/14783363.2017.1413344

Camilleri, M. A. (2021a). Sustainable Production and Consumption of Food. Mise-en-Place Circular Economy Policies and Waste Management Practices in Tourism Cities, Sustainability, 13(17), 9986.

Camilleri, M. A. (2021b). Strategic attributions of corporate social responsibility and environmental management: The business case for doing well by doing good!. Sustainable Development. https://doi.org/10.1002/sd.2256 
Camilleri, M.A. (2021c). The market for socially responsible investing: a review of the developments. Social Responsibility Journal, 17(3), 412-428. https://doi.org/10.1108/SRJ-06-20190194

Carvalho, F., Santos, G., \& Gonçalves, J. (2020). Critical analysis of information about integrated management systems and environmental policy on the Portuguese firms' website, towards sustainable development. Corporate Social Responsibility and Environmental Management, 27(2), 1069-1088.

Chiarini, A. (2019). Factors for succeeding in ISO 14001 implementation in Italian construction industry. Business Strategy and the Environment, 28(5), 794-803.

Colwell, S. R., \& Joshi, A. W. (2013). Corporate ecological responsiveness: Antecedent effects of institutional pressure and top management commitment and their impact on organizational performance. Business Strategy and the Environment, 22(2), 73-91.

Clemens, B. W., \& Douglas, T. J. (2005). Understanding strategic responses to institutional pressures. Journal of Business Research, 58(9), 1205-1213.

Curkovic, S., \& Sroufe, R. (2011). Using ISO 14001 to promote a sustainable supply chain strategy. Business Strategy and the Environment, 20(2), 71-93.

Da Fonseca, L. M. C. M. (2015). ISO 14001: 2015: An improved tool for sustainability. Journal of Industrial Engineering and Management, 8(1), 37-50.

Darnall, N. (2006). Why firms mandate ISO 14001 certification. Business \& Society, 45(3), 354-381.

Delmas, M. (2001). Stakeholders and competitive advantage: the case of ISO 14001. Production and Operations Management, 10(3), 343-358.

Delmas, M. A. (2002). The diffusion of environmental management standards in Europe and in the United States: An institutional perspective. Policy Sciences, 35(1), 91-119.

Delmas, M. A., \& Toffel, M. W. (2008). Organizational responses to environmental demands: Opening the black box. Strategic Management Journal, 29(10), 1027-1055.

Demuijnck, G., \& Fasterling, B. (2016). The social license to operate. Journal of Business Ethics, 136(4), 675-685.

Dissou, Y. (2005). Cost-effectiveness of the performance standard system to reduce CO2 emissions in Canada: a general equilibrium analysis. Resource and Energy Economics, 27(3), 187207.

Duthler, G., \& Dhanesh, G. S. (2018). The role of corporate social responsibility (CSR) and internal CSR communication in predicting employee engagement: Perspectives from the United Arab Emirates (UAE). Public Relations Review, 44(4), 453-462. 
Eltayeb, T. K., Zailani, S., \& Ramayah, T. (2011). Green supply chain initiatives among certified companies in Malaysia and environmental sustainability: Investigating the outcomes. Resources, Conservation and Recycling, 55(5), 495-506.

Erauskin-Tolosa, A., Zubeltzu-Jaka, E., Heras-Saizarbitoria, I., \& Boiral, O. (2020). ISO 14001, EMAS and environmental performance: A meta-analysis. Business Strategy and the Environment, 29(3), 1145-1159.

Ettinger, A., Grabner-Kräuter, S., \& Terlutter, R. (2018). Online CSR communication in the hotel industry: Evidence from small hotels. International Journal of Hospitality Management, 68, 94-104.

EU (2014). Directive 2014/95/EU of the European Parliament and of the Council of 22 October 2014 amending Directive 2013/34/EU as regards disclosure of non-financial and diversity information by certain large undertakings and groups. European Commission, Brussels, Belgium. https://eur-lex.europa.eu/legal-content/EN/TXT/?uri=CELEX\%3A32014L0095 (accessed 05 January 2022).

EU (2019). Regulation of the European Parliament and of the council amending Regulation (EU) 2019/631 as regards strengthening the CO2 emission performance standards for new passenger cars and new light commercial vehicles in line with the Union's increased climate ambition. 2021. European Commission. Brussels, Belgium. Available online: https://ec.europa.eu/info/sites/default/files/amendment-regulation-co2-emission-standards-cars-vanswith-annexes_en.pdf (accessed 05 October 2021).

EU (2021). EU Taxonomy, Corporate Sustainability Reporting, Sustainability Preferences and Fiduciary Duties: Directing finance towards the European Green Deal COM/2021/188 final European Commission. Brussels, Belgium. Available online: https://eur-lex.europa.eu/legalcontent/EN/TXT/?uri=CELEX:52021DC0188 (accessed 05 January 2022).

Fatica, S., \& Panzica, R. (2021). Green bonds as a tool against climate change?. Business Strategy and the Environment, 30(5), 2688-2701.

Fortuna, F., Testarmata, S., Sergiacomi, S., \& Ciaburri, M. (2020). Mandatory disclosure of non-financial information: A structured literature review. In Mara Del Baldo, Jesse Dillard, Maria-Gabriella Baldarelli, Massimo Ciambotti (Eds.), Accounting, Accountability and Society, 95-128, Springer, Cham, Switzerland.

Garrido, E., González, C., \& Orcos, R. (2020). ISO 14001 and CO2 emissions: An analysis of the contingent role of country features. Business Strategy and the Environment, 29(2), 698710 .

Gatto, A. (2020). A pluralistic approach to economic and business sustainability: A critical meta-synthesis of foundations, metrics, and evidence of human and local development. Corporate Social Responsibility and Environmental Management, 27(4), 1525-1539.

Gerged, A. M., Matthews, L., \& Elheddad, M. (2021). Mandatory disclosure, greenhouse gas emissions and the cost of equity capital: UK evidence of a U-shaped relationship, Business Strategy and the Environment, 30(2), 908-930. 
Govindan, K., Khodaverdi, R., \& Vafadarnikjoo, A. (2015). Intuitionistic fuzzy based DEMATEL method for developing green practices and performances in a green supply chain. Expert Systems with Applications, 42(20), 7207-7220.

Heras-Saizarbitoria, I., Arana, G., \& Boiral, O. (2016). Outcomes of environmental management systems: The role of motivations and firms' characteristics. Business Strategy and the Environment, 25(8), 545-559.

Heras-Saizarbitoria, I., \& Boiral, O. (2013). ISO 9001 and ISO 14001: towards a research agenda on management system standards. International Journal of Management Reviews, 15(1), 47-65.

Hillary, R. (2004). Environmental management systems and the smaller enterprise. Journal of Cleaner Production, 12(6), 561-569.

Hojnik, J., Ruzzier, M., \& Manolova, T. S. (2018). Internationalization and economic performance: The mediating role of eco-innovation. Journal of Cleaner Production, 171, 1312-1323.

Hsu, C. C., Tan, K. C., Zailani, S. H. M., \& Jayaraman, V. (2013). Supply chain drivers that foster the development of green initiatives in an emerging economy. International Journal of Operations \& Production Management, 36(1), 86-110.

Husted, B. W., Montiel, I., \& Christmann, P. (2016). Effects of local legitimacy on certification decisions to global and national CSR standards by multinational subsidiaries and domestic firms. Journal of International Business Studies, 47(3), 382-397.

Iatridis, K., \& Kesidou, E. (2018). What drives substantive versus symbolic implementation of ISO 14001 in a time of economic crisis? Insights from Greek manufacturing companies. Journal of Business Ethics, 148(4), 859-877.

Ikram, M., Mahmoudi, A., Shah, S. Z. A., \& Mohsin, M. (2019). Forecasting number of ISO 14001 certifications of selected countries: application of even GM $(1,1)$, DGM, and NDGM models. Environmental Science and Pollution Research, 26(12), 12505-12521.

Ikram, M., Zhang, Q., Sroufe, R., \& Shah, S. Z. A. (2020). Towards a sustainable environment: The nexus between ISO 14001, renewable energy consumption, access to electricity, agriculture and CO2 emissions in SAARC countries. Sustainable Production and Consumption, 22, 218-230.

ISO 2021. ISO Survey of certifications to management system standards - Full results, 2021. International Standards Organization, Geneva, Switzerland. Available from: https://isotc.iso.org/livelink/livelink?func=ll\&objId=18808772\&objAc$\underline{\text { tion=browse \& view Type }=1}$ (accessed on 05 October 2021).

Jabbour, C. J. C. (2015). Environmental training and environmental management maturity of Brazilian companies with ISO14001: empirical evidence. Journal of Cleaner Production, 96, 331-338. 
Jabbour, C.J.C., Seuring, S., de Sousa Jabbour, A. B. L., Jugend, D., Fiorini, P. D. C., Latan, H., \& Izeppi, W. C. (2020). Stakeholders, innovative business models for the circular economy and sustainable performance of firms in an emerging economy facing institutional voids. Journal of Environmental Management, 264, https://doi.org/10.1016/j.jenvman.2020.110416 (accessed on 08 January 2022).

Jacobs, B. W., Singhal, V. R., \& Subramanian, R. (2010). An empirical investigation of environmental performance and the market value of the firm. Journal of Operations Management, 28(5), 430-441.

Khan, S. Z., Yang, Q., \& Waheed, A. (2019). Investment in intangible resources and capabilities spurs sustainable competitive advantage and firm performance. Corporate Social Responsibility and Environmental Management, 26(2), 285-295.

Kitazawa, S., \& Sarkis, J. (2000). The relationship between ISO 14001 and continuous source reduction programs. International Journal of Operations \& Production Management, 20(2), $225-248$.

Klassen, R. D., \& Vachon, S. (2003). Collaboration and evaluation in the supply chain: The impact on plant-level environmental investment. Production and Operations Management, 12(3), 336-352.

Kong, D., Liu, S., \& Dai, Y. (2014). Environmental policy, company environment protection, and stock market performance: Evidence from China. Corporate Social Responsibility and Environmental Management, 21(2), 100-112.

Laosirihongthong, T., Adebanjo, D., \& Tan, K. C. (2013). Green supply chain management practices and performance. Industrial Management \& Data Systems, 113(8), 1088-1109.

Latan, H., Jabbour, C. J. C., de Sousa Jabbour, A. B. L., Wamba, S. F., \& Shahbaz, M. (2018). Effects of environmental strategy, environmental uncertainty and top management's commitment on corporate environmental performance: The role of environmental management accounting, Journal of Cleaner Production, 180, 297-306.

Lee, S. M., Noh, Y., Choi, D., \& Rha, J. S. (2017). Environmental policy performances for sustainable development: from the perspective of ISO 14001 certification. Corporate Social Responsibility and Environmental Management, 24(2), 108-120.

Ljungberg, L. Y. (2007). Materials selection and design for development of sustainable products. Materials \& Design, 28(2), 466-479.

Ma, Y., Liu, Y., Appolloni, A., \& Liu, J. (2021). Does green public procurement encourage firm's environmental certification practice? The mediation role of top management support. Corporate Social Responsibility and Environmental Management, 28(3), 1002-1017.

Martín-de Castro, G., Amores-Salvadó, J., \& Navas-López, J. E. (2016). Environmental management systems and firm performance: Improving firm environmental policy through stakeholder engagement. Corporate Social Responsibility and Environmental Management, 23(4), 243-256. 
Melnyk, S. A., Sroufe, R. P., \& Calantone, R. (2003). Assessing the impact of environmental management systems on corporate and environmental performance. Journal of Operations Management, 21(3), 329-351.

Miroshnychenko, I., Barontini, R., \& Testa, F. (2017). Green practices and financial performance: A global outlook. Journal of Cleaner Production, 147, 340-351.

Moher, D., Shamseer, L., Clarke, M., Ghersi, D., Liberati, A., Petticrew, M., ... \& Stewart, L. A. (2015). Preferred reporting items for systematic review and meta-analysis protocols (PRISMA-P) 2015 statement. Systematic Reviews, 4(1), 1-9.

Mtapuri, O., Camilleri, M. A., \& Dłużewska, A. (2021). Advancing community-based tourism approaches for the sustainable development of destinations. Sustainable Development, https://doi.org/10.1002/sd.2257

Nakamura, M., Takahashi, T., \& Vertinsky, I. (2001). Why Japanese firms choose to certify: a study of managerial responses to environmental issues. Journal of Environmental Economics and Management, 42(1), 23-52.

Ntim, C. G. \& Soobaroyen, T. (2013). Corporate governance and performance in socially responsible corporations: new empirical insights from a neo-institutional framework. Corporate Governance, 21(5), 468-494.

Nunhes, T. V., Motta, L. C. F., \& de Oliveira, O. J. (2016). Evolution of integrated management systems research on the Journal of Cleaner Production: Identification of contributions and gaps in the literature. Journal of Cleaner Production, 139, 1234-1244.

Oliveira, J. A., Oliveira, O. J., Ometto, A. R., Ferraudo, A. S., \& Salgado, M. H. (2016). Environmental Management System ISO 14001 factors for promoting the adoption of Cleaner Production practices. Journal of Cleaner Production, 133, 1384-1394.

Orsato, R. J. (2006). Competitive environmental strategies: when does it pay to be green? California Management Review, 48(2), 127-143.

Palea, V., \& Drogo, F. (2020). Carbon emissions and the cost of debt in the eurozone: The role of public policies, climate-related disclosure and corporate governance. Business Strategy and the Environment, 29(8), 2953-2972.

Para-González, L., \& Mascaraque-Ramírez, C. (2019). The importance of official certifications in globalized companies' performance: An empirical approach to the shipbuilding industry. Corporate Social Responsibility and Environmental Management, 26(2), 408-415.

Park, E. (2019). Corporate social responsibility as a determinant of corporate reputation in the airline industry. Journal of Retailing and Consumer Services, 47, 215-221.

Potoski, M., \& Prakash, A. (2005). Green clubs and voluntary governance: ISO 14001 and firms' regulatory compliance. American Journal of Political Science, 49(2), 235-248. 
Potoski, M., \& Prakash, A. (2005). Covenants with weak swords: ISO 14001 and facilities' environmental performance. Journal of Policy Analysis and Management: The Journal of the Association for Public Policy Analysis and Management, 24(4), 745-769.

Prakash, A., \& Potoski, M. (2006). The voluntary environmentalists: Green clubs, ISO 14001, and voluntary environmental regulations. Cambridge University Press, Cambridge, UK.

Morrow, D., \& Rondinelli, D. (2002). Adopting corporate environmental management systems:: Motivations and results of ISO 14001 and EMAS certification. European Management Journal, 20(2), 159-171.

Rennings, K., Ziegler, A., Ankele, K., \& Hoffmann, E. (2006). The influence of different characteristics of the EU environmental management and auditing scheme on technical environmental innovations and economic performance. Ecological Economics, 57(1), 45-59.

Riaz, H., \& Saeed, A. (2020). Impact of environmental policy on firm's market performance: The case of ISO 14001. Corporate Social Responsibility and Environmental Management, 27(2), 681-693.

Rivera-Arrubla, Y. A., Zorio-Grima, A., \& García-Benau, M. A. (2017). Integrated reports: disclosure level and explanatory factors. Social Responsibility Journal, 13, 1, 155-176.

Robèrt, K. H., Schmidt-Bleek, B., De Larderel, J. A., Basile, G., Jansen, J. L., Kuehr, R., ... \& Wackernagel, M. (2002). Strategic sustainable development—selection, design and synergies of applied tools. Journal of Cleaner Production, 10(3), 197-214.

Romito, S., \& Vurro, C. (2021). Non-financial disclosure and information asymmetry: A stakeholder view on US listed firms. Corporate Social Responsibility and Environmental Management, 28(2), 595-605.

Russo, M. V. (2009). Explaining the impact of ISO 14001 on emission performance: a dynamic capabilities perspective on process and learning. Business Strategy and the Environment, 18(5), 307-319.

Salim, H. K., Padfield, R., Hansen, S. B., Mohamad, S. E., Yuzir, A., Syayuti, K., ... \& Papargyropoulou, E. (2018). Global trends in environmental management system and ISO14001 research. Journal of Cleaner Production, 170, 645-653.

Salomone, R. (2008). Integrated management systems: experiences in Italian organizations. Journal of Cleaner Production, 16(16), 1786-1806.

Santos, G., Rebelo, M., Lopes, N., Alves, M. R., \& Silva, R. (2016). Implementing and certifying ISO 14001 in Portugal: motives, difficulties and benefits after ISO 9001 certification. Total Quality Management \& Business Excellence, 27(11-12), 1211-1223.

Scur, G., \& Barbosa, M. E. (2017). Green supply chain management practices: Multiple case studies in the Brazilian home appliance industry. Journal of Cleaner Production, 141, 12931302. 
Secinaro, S., Brescia, V., Calandra, D., \& Saiti, B. (2020). Impact of climate change mitigation policies on corporate financial performance: Evidence-based on European publicly listed firms. Corporate Social Responsibility and Environmental Management, 27(6), 2491-2501.

Shaharudin, M. R., Govindan, K., Zailani, S., Tan, K. C., \& Iranmanesh, M. (2017). Product return management: Linking product returns, closed-loop supply chain activities and the effectiveness of the reverse supply chains. Journal of Cleaner Production, 149, 1144-1156.

Soewarno, N., Tjahjadi, B., \& Fithrianti, F. (2019). Green innovation strategy and green innovation: The roles of green organizational identity and environmental organizational legitimacy. Management Decision, 57(11), 3061-3078.

Souza, J. P. E., \& Alves, J. M. (2018). Lean-integrated management system: A model for sustainability improvement. Journal of Cleaner Production, 172, 2667-2682.

Su, H. C., Dhanorkar, S., \& Linderman, K. (2015). A competitive advantage from the implementation timing of ISO management standards. Journal of Operations Management, 37, 3144.

Su, L., Swanson, S. R., \& Chen, X. (2018). Reputation, subjective well-being, and environmental responsibility: The role of satisfaction and identification. Journal of Sustainable Tourism, 26(8), 1344-1361.

Teng, M. J., \& Wu, S. Y. (2018). Sustainable development and competitive advantages-utilizing matching to overcome sample selection bias. Corporate Social Responsibility and Environmental Management, 25(4), 313-326.

Testa, F., Boiral, O., \& Iraldo, F. (2018). Internalization of environmental practices and institutional complexity: Can stakeholders pressures encourage greenwashing?. Journal of Business Ethics, 147(2), 287-307.

Testa, F., Rizzi, F., Daddi, T., Gusmerotti, N. M., Frey, M., \& Iraldo, F. (2014). EMAS and ISO 14001: the differences in effectively improving environmental performance. Journal of Cleaner Production, 68, 165-173.

Teixeira, A. A., Jabbour, C. J. C., de Sousa Jabbour, A. B. L., Latan, H., \& De Oliveira, J. H. C. (2016). Green training and green supply chain management: evidence from Brazilian firms. Journal of Cleaner Production, 116, 170-176.

UNFCC (2021). COP26 Reaches Consensus on Key Actions to Address Climate Change. United Nations Climate Change, Bonn, Germany. https://unfccc.int/news/cop26-reaches-consensus-on-key-actions-to-address-climate-change (accessed on 08 January 2022).

Vijayvargy, L., Thakkar, J., \& Agarwal, G. (2017). Green supply chain management practices and performance: the role of firm-size for emerging economies. Journal of Manufacturing Technology Management, 28(3), 299-323.

Weber, O. (2014). Environmental, social and governance reporting in China. Business Strategy and the Environment, 23(5), 303-317. 
Wilburn, K. M., \& Wilburn, R. (2011). Achieving social license to operate using stakeholder theory. Journal of International Business Ethics, 4(2), 3-16.

Xu, X. D., Zeng, S. X., Zou, H. L., \& Shi, J. J. (2016). The impact of corporate environmental violation on shareholders' wealth: A perspective taken from media coverage. Business Strategy and the Environment, 25(2), 73-91.

Yu, H. C., Kuo, L., \& Kao, M. F. (2017). The relationship between CSR disclosure and competitive advantage. Sustainability Accounting, Management and Policy Journal, 8(5), 547-570.

Zailani, S. H. M., Eltayeb, T. K., Hsu, C. C., \& Tan, K. C. (2012). The impact of external institutional drivers and internal strategy on environmental performance. International Journal of Operations \& Production Management, 32(6),721-745.

Zorpas, A. A. (2020). Strategy development in the framework of waste management. Science of the Total Environment, 716, Available from: https://doi.org/10.1016/j.scitotenv.2020.137088 (accessed on 05 October 2021). 
